\title{
TWO CASES
}

\section{OF \\ PYLORECTOMY AND ONE OF JEJUNOSTOMY, \\ WITH REMARKS,}

AND WITH A SUGGESTED MODIFICATION OF THE

FORMER OPERATION.

BY

A. W. MAYO ROBSON, F.R.C.S.,

PROFRGSOR OF SURGERY IN THE VICTORIA UNIVERSITY AND HOX.

SURGEON IN THF LEEDS GENERAI INPIRMARY.

Received January 5th-Read June 14th, 1892.

I AM bringing forward the following cases for two reasons: 1st, because it seems to me important that all serious and especially comparatively new operative procedures should be chronicled for statistical and comparative purposes; 2nd, because it is not yet clearly established that these operations for cancer can be relied on to produce such an amount of relief as to warrant their continuance.

Concerning the operation of gastrostomy for the prolongation of life in impermeable cancer of the œsophagus, and gastro-enterostomy for pyloric obstruction, I think there is sufficient evidence to prove that the relief 
afforded is amply sufficient to warrant the continuance of their practice, and I will not spend the time of the Society by detailing cases, as they have been discussed fully here and elsewhere; and, moreover, I have myself written on both subjects in the 'British Medical Journal' for June 7th, 1890, and the 'Lancet' for May 23rd, 1891. I think, however, that I ought to mention a sequence of gastroenterostomy which has twice occurred in my practice, and which, so far as I am aware, has not been previously mentioned; it is the regurgitation of the intestinal contents into the stomach through the artificial opening, interfering with assimilation and producing offensive eructation and nausea. The three following cases are given in detail :

CASE 1. Pylorectomy for pyloric stenosis due to cancer; recovery; recurrence of symptoms due to contraction of the cicatrix ; gastro-enterostomy four months after ; regurgitation of intestinal contents and fæcal poisoning.-W. S-, æt. 54, a cabinet-maker, was admitted to the Infirmary on June 23rd, 1891, when he gave the history that he had been quite well up to six months previously, when he began to lose his appetite and to experience a feeling of weight at the stomach after food. This was soon followed by pain in the epigastrium, with occasional vomiting of the stomach contents. During the past two months the vomit had been dark-coloured, and had occurred almost constantly after food. Beyond a general fulness of the abdomen he had not noticed any localised swelling. During the eight months he had lost about two stone in weight.

On admission the patient was found to be much emaciated, and on examination of the abdomen well-marked signs of distension of the stomach were discovered. A tumour about the size of a walnut could be felt an inch to the right of the middle line and an inch and a half above the umbilicus; this was rather painful on pressure, and at some times was much more distinctly felt than at others. 
The tumour, which was dull on percussion, was not fixed, and moved up and down with respiration.

Vomiting occurred after every meal, the vomit consisting of almost unaltered food mixed with coffee-ground material, and on examination blood was found to be present and free hydrochloric acid absent. A diagnosis of cancer of the pylorus was made, and pylorectomy was decided on after consultation.

Liquid food only was administered on June 24th, and after dinner all food was stopped by the mouth, nutrient injections being given. In the evening the upper part of the abdomen was shaved, an antiseptic dressing applied, and the stomach washed out with a solution of boroglyceride; the stomach lavage was repeated on the morning of the 25th, and again half an hour before the operation, which was performed at 2 p.m. on June 25th, the A. C. E. mixture being the anæsthetic.

An incision of three inches was made in the linea alba above the umbilicus, and after a few peritoneal adhesions had been broken down, the pyloric tumour was easily brought to the surface; the disease was apparently limited to the pylorus, extending only a small distance along the anterior wall of the stomach. A handled needle with a slit on its convex side was pushed through the gastro-hepatic omentum above the pylorus; the loop of a silk ligature was introduced into the slit and the needle withdrawn, thus leaving, after division of the loop, two ligatures in situ, which were then tightened, the gastro-hepatic omentum being divided between the two; this process was repeated on the right and left of the first ligatures, thus separating the pylorus above. The index finger of the left hand was then passed through this opening into the lesser cavity of the peritoneum, and a similar procedure was adopted in the division of the great omentum, no blood being lost in the process.

Hahn's clamps were then applied to the stomach and duodenum beyond the disease, which was then removed by means of scissors. After all bleeding points had been 
ligatured, the wound in the stomach was closed by a continuous suture, and made secure by Lembert's sutures from above downwards, leaving an opening at the lower end equal in size to the opening in the duodenum.

The peritoneum of the posterior halves of the openings in the stomach and duodenum was first brought together by Lembert's sutures; the mucous membrane of the stomach and duodenum were then connected all round, and lastly, the anterior halves of the stomach and duodenal openings were connected by Lembert's sutures. As sponges had been packed around the site of operation, the peritoneum had not become in any way fouled, and practically no blood was lost. The parietal wound was then closed in the ordinary way by six silkworm gut sutures. The whole operation occupied an hour and a quarter. The after progress was all that could be desired, the temperature never exceeding the normal, and no shock being

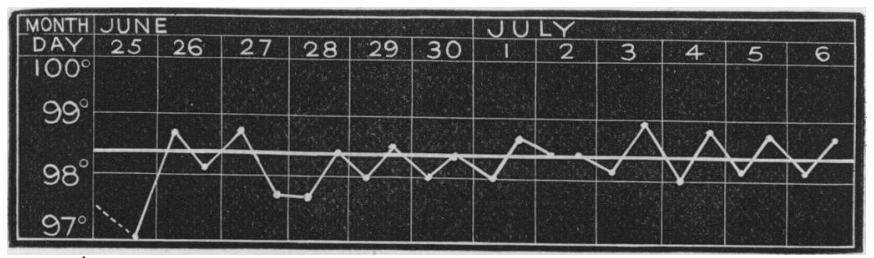

manifested. During the first twenty-four hours there was a little vomiting of dark-coloured fluid ; on the second day Brand's essence and barley water were given; on the third day milk and soda water and beef tea were administered; on the fourth day the patient took about three pints of milk, with Brand's essence and other light. food.

On July 4th, i.e. a week after the operation, the wound was dressed for the first time and found to be healed, the stitches being removed. The patient was then taking fish and rice pudding; the next day he had chicken; and the following day he took a mutton chop with good relish, and digested it. He rapidly regained strength and flesh, 
and was made an out-patient within a month of the operation, expressing himself and feeling and looking well.

The tumour removed was about the size of a large walnut, and hard both to touch and on section. The peritoneal surface was smooth and apparently unaffected.

A clear margin of healthy tissue intervened between the growth and the cut surface.

The mucous surface was dark and ulcerated.

The pyloric orifice was much constricted, admitting when relaxed the tip of the little finger.

August 21st, 1891.-The patient came to the infirmary to report that his vomiting had returned, and that he was losing the flesh he had regained. The vomit consisted of fermented food, and was entirely free from the coffeeground character which had previously been present. It contained stringy mucus and yeast-cells, but no sarcinæ and no free hydrochloric acid; the vomiting came on half an hour to two hours after food. There were manifest signs of dilatation of the stomach, but no tumour or other abnormality could be felt. Although the stomach was washed out daily he rapidly lost flesh, and on September 8th a note was made to the effect that he had lost nine and a half pounds since admission. On September 9th it was manifest the patient was rapidly running down, and he was so weak that it was felt inadrisable to continue the lavage, and questionable whether he was strong enough to bear any further operative procedure; but as it was now evident that the pyloric opening had again contracted the state of affairs was explained to him and his wife, and the operation of gastro-enterostomy was proposed.

On September 10th, ether being the anæsthetic, the abdomen was opened in the line of the old scar. So far as could be discovered there was no return of the disease at the pylorus, and the parts appeared quite natural except for the dilatation of the stomach. A loop of the jejunum was easily found by following it down from its fixed point on the left of the spine; this was then emptied and rendered bloodless by drawing it between the fingers of the 
left hand, and encircling its base by a tourniquet composed of a piece of elastic tubing, which was tied in a single knot and clamped by pressure forceps. Senn's bone-plates were then introduced through incisions in the bowel and the lower part of the stomach, and were fixed in the usual way, four Lembert's sutures being introduced around the plates for greater security.

The peritoneum was not soiled in any way, and the abdomen was closed by silkworm gut sutures. The operation occupied three quarters of an hour. The patient rallied well from the operation, although in the evening his temperature was only $97 \cdot 2^{\circ}$.

The nutrient injections were continued every four hours, and a teaspoonful of Valentine's meat juice was given every hour. On September 11th the patient seemed better, and had neither vomiting nor pain, but the meat juice had to be stopped for a few hours, as it made him sickly. Temperature normal. Pulse weak. On September 12th, beyond the weakness, the patient seemed fairly well, being entirely free from pain or sickness. His breath, however, had an unpleasant odour, and the nutrient enemata were not retained, but brandy and liquid food was given by the mouth and retained. On September 13th, without any new symptoms developing, the patient became gradually weaker, and died exhausted in the afternoon. I have to thank my colleague Dr. Barrs, Honorary Pathologist, for the following report :

Post-mortem examination made September 14th:" Extremely emaciated body, scarcely a particle of fat anywhere, slaty-black staining of peritoneal surfaces, probably due to blood-staining of operation in June. No general peritonitis. Some old adhesions in pyloric region. Recent adhesion of loop of jejunum to pyloric end of stomach, embedding Lembert's sutures. It may be said the operation of gastro-enterostomy had been quite satisfactory, the highest part of the jejunum available had been opened into the lowest possible point of the stomach, 
and everything was in good order. The intestinal plate was partly eroded, the stomach plate was loosened and softened, and the ligatures of both were still in situ. There was a patent and sufficiently capacious opening between the two viscera. The stomach contained some grumous, fæcal-looking fluid, and the mucous membrane was stained in such a manner as to strongly suggest that the intestinal contents had passed into the stomach. The gastric musculosa and mucosa were much hypertrophied. The pyloric orifice would not admit the tip of the little finger without force, the natural pyloric ring being replaced by a sharp, tight, cord-like cicatrix. Externally the parts had an almost natural appearance, and there was no sign of new growth in the stomach or in any other organs. All the other organs were healthy.

CASE 2.-Mr. T. B-, æt. 57, called to consult me on the 10th of June, 1891, on account of dyspeptic symptoms accompanied by vomiting and great loss of flesh. He told me that he had been well up to October, 1890, when he began to have discomfort at the pit of the stomach, with a want of appetite and sickness, but no vomiting since Christmas. He had been treated for indigestion, and had been taking medicines of various kinds without benefit, and during the six months previous to my seeing him he had lost $22 \mathrm{lbs}$. in weight. During May and June he had vomited every second or third day. When I saw him there were manifest signs of dilatation of the stomach, and a tumour about the size of a hen's egg could be felt midway between the umbilicus and the right costal margin. His immediate symptoms were relieved by washing out the stomach, and by the administration of salicylate of soda and strychnia. The pain disappeared, the vomiting ceased, and he was less troulled with flatulency and constipation. For a fortnight he ceased to lose weight, but without any corresponding increase in strength. My diagnosis was cancer of the pylorus, and this I explained to Mr. B- 
and his friends, telling him at the time that I saw no chance of relief except by operation.

On July 2nd Dr. Barrs saw the patient with me, and agreed as to the advisability of operating.

On July 7th pylorectomy was performed as in the last case, but as the tumour extended for some distance along the duodenum the resection was more extensive, and it was necessary to remove an extra portion from the posterior wall of the duodenum, as it was found that the section was made quite close to the growth. Two enlarged glands were also removed. No special difficulty was experienced in bringing the parts together, and very little blood was lost. The operation occupied one hour and a half. The patient rallied well, experienced very little shock, and only vomited once. Barley water in teaspoonful doses was given during the first twenty-four hours, after which Valentine's meat juice, Brand's essence, and peptonised milk and soda water with a little brandy were given. Nutrient injections were also administered, but unfortunately were never retained.

The patient appeared to be doing fairly well up to the third day, except that he did not regain strength, and from the evening of the third day until the termination of the case he simply gradually got weaker, and died of exhaustion on the morning of the fifth day.

So far as could be ascertained in the absence of a postmortem examination, death appeared to be due simply to exhaustion, and not to any complication of the operation, as there was an entire absence of distension, vomiting, or other sign of peritonitis. The resected tumour proved to be carcinoma, which had almost obliterated the lumen of the pylorus, so that it would only admit the passage of a No. 4 catheter.

CASE 3. Extensive cancer of stomach, with inability to retain food; jejunostomy; recovery from operation; death from progress of disease at end of two months.-Mrs. E. B-, æt. 58, residing in Lincolnshire, was sent to me on June 
19th, 1891, by Dr. Hamilton, of Crowle, on account of an abdominal tumour, accompanied by persistent vomiting and rapid loss of flesh. The patient said that she had been quite well until a year and a half before admission, when she was suddenly attacked with vomiting, which had continued ever since. She had lost weight from that time until, on admission, she was worn almost to a skeleton. She had never had hæmatemesis. The vomiting took place directly after food, and was unaccompanied by nausea. At the beginning she had a stabbing pain after food in the region of the left breast, but during the six months before admission the pain had been continuous, and always worse after eating. About three months before admission she first noticed a swelling in the left hypochondrium, which had increased rapidly up to the time of admission. From the time the tumour was noticed the pain always seemed to radiate from it.

On admission the patient was extremely emaciated, and vomited everything immediately after eating. The vomit consisted simply of what she had taken, and contained free hydrochloric acid, but no sarcinæ or bloodcells could be detected microscopically. Occupying the left hypochondrium and reaching into the epigastrium was a hard nodular tumour, moving up and down with respiration, the skin being quite movable over it. A tumour could also be felt beneath the liver, which was diagnosed as a distended gall-bladder.

Cancer of the stomach was diagnosed, and the patient was fed with small quantities of Brand's essence and peptonised foods, nutrient enemata being also administered. The pain was controlled by morphia.

At first slight improvement took place, but towards the end of the month she again lost ground, and the vomiting still persisted. After consultation an exploratory incision was decided on, in order to see if the disease could be removed; but if it should prove too extensive for removal, as it was suspected it might, jejunostomy could be done.

On July 1st, 1891, the A. C. E. mixture being the anæs- 
thetic, and the skin of the abdomen having been asepticised, an incision of three inches was made in the linea alba above the umbilicus, exposing the tumour, which was found to be occupying the whole of the anterior wall of the stomach, and therefore incapable of removal. There was also a distended gall-bladder, containing gallstones.

The jejunum was then found at its fixed point on the left of the spine, and traced for about six inches downwards, at which spot a knuckle of the bowel was brought forward and fixed by loop sutures (after the parietal peritoneum and skin had been connected by a continuous suture) in the way suggested for gastrostomy by $\mathbf{M r}$. Greig Smith, and which a case of mine published in the 'British Medical Journal' for June 7th, 1890, proves to. be a safe and efficient method.

A loop of silver wire was first inserted into the convex surface of the bowel at a spot where it would have to be opened. A round, large-eyed sewing needle was then threaded with twelve inches of silk of medium thickness, and passed under the peritoneal coat of the intestine in a circle about one and a half inches in diameter, the suture being made to emerge and leave five loops at equal intervals. The loops were drawn through the parietes at about one third of an inch from the margin of the wound. As each loop was drawn up a piece of No. 6 catheter was passed through it, and when all were in position the ends of the silk were drawn in and the loops tightened over the catheter, over which the two ends were then knotted; the silver wire was then fixed under the catheter, and a few sutures connected the bowel to the skin. The rest of the parietal incision was brought together by interrupted silkworm gut sutures in the ordinary way.

On the following day the patient felt quite comfortable, and was fed by the rectum entirely. This was continued until July 7th, when a small opening was made in the exposed bowel with a tenotomy knife, and a soft catheter was passed in, through which she was fed by peptonised food, 
of which she was able to take two or three pints a day. She gained flesh, and was able to return home on July 27th. Her chief trouble was that the skin around the artificial opening became irritable ; but through the opening into the duodenum she continued to be able to take a fair quantity of food, which was well retained. The original disease continued to advance, and after two months she died from exhaustion.

No autopsy could be obtained.

Remarks. - With regard to the operation of jejunostomy, my experience in Case 3 would lead me to conclude that life can undoubtedly be prolonged by it in cases of cancer of the stomach too extensive for removal, and when food cannot be taken; but it seems to me that it ought to be clearly stated beforehand to the patient that relief only will occur, and that existence under the circumstances may not be particularly comfortable, although life may actually be prolonged for weeks, or even for months. An opening into the jejunum for feeding purposes is quite different from an opening into the stomach, and is not nearly so satisfactory, as the edges of the fistula tend to become irritated by bile and intestinal secretion, producing considerable discomfort.

In cancer of the pylorus, pylorectomy undoubtedly offers a chance of cure and a possibility of considerable relief, but for the operation to be successful it must be done at quite an early stage. In my second case, although the disease was to all appearances in quite an early stage, it had already involved the glands, and invaded the contiguous parts of the stomach and duodenum.

It would seem to me preferable not to be wedded to any special operation in these cases, but at the time of exploration to be guided by circumstances : for instance, where the disease is very extensive, having become fixed and having involved glands, I should prefer gastro-enterostomy to any form of pylorectomy.

Where the disease is fairly advanced, yet not too exvol. LXXXv. 
tensive to prevent successful removal, I would suggest pylorectomy with closure of the cut ends of the stomach and duodenum, and immediate gastro-enterostomy, as in the operation performed by Mr. Lawson, Mr. Greig Smith, Dr. Bull, and Mr. Jessett.

Where, however, the disease is taken early, is not too extensive, has not involved glands, and has not fixed the pylorus to the contiguous parts, I should decidedly prefer simple pylorectomy, as in the cases I have reported ; but in order to avoid the occurrence of cicatricial constriction I shall in future use a decalcified bone tube almost equal in diameter to the cut section of the duodenum, the tube being about an inch in length, with an elevated rim at each end (rather like a cotton bobbin) to prevent its displacement at too early a stage. This method occurred to we when I was operating on my first case, but as the patient had recovered and appeared so well at the time I performed the second operation, I did not feel it necessary to vary un procedure and carry out the idea, but I shall certainly carry it out in future, as I think it will give greater security at the time and prevent after contraction.

By means of the tube, and by using a continuous suture to the margins, of the openings and another continuous suture applied so as to bring the serous surfaces into apposition one third of an inch from the margins, the operation can be shortened very considerably, and on the cadaver I have performed it in quite a short time.

The diagrams shown will, I trust, serve to make plain the method of pylorectomy I suggest, and the models will, I trust, further illustrate my suggestions.

With a little modification I am employing the same tube in gastro-enterostomy, in enterectomy, and in intestinal anastomosis.

\section{ADDENDUM.}

The modification of pylorectomy by the employment of a cotton-reel shaped tube, though simple, is difficult to 
explain without showing the tubes and illustrating their use by diagrams and models; I hope, however, that by their help I can make clear how the operation is done.

The tubes, made of decalcified bone, are of various sizes, and secure an immediate communication between the two apposed hollow viscera. The raised ends of the tube prevent the appliance slipping either into the stomach or duodenum until union is accomplished between them. Of the two continuous sutures, one is marginal, and when drawn tight causes the edges of the openings in the stomach and duodenum to be firmly applied to the tube, thus preventing extravasation of visceral contents.

The second continuous suture, placed one third or half an inch from the margin of the opening, takes up peritoneum only, and secures peritoneal apposition for fully one third of an inch from the visceral opening all round. No other sutures are required, hence the operation can be quickly performed, especially after a little practice on models or on the cadaver.

The tube can be varied in size according to the diameter of the cut section of the bowel. By securing a continuous mucous surface through the opening, a narrowing of the artificial channel will not be likely to occur ; moreover the communication between any two viscera may be made as large or as small as required, according to the size of the tube employed. The method is equally available for pylorectomy, for end-to-end enterorrhaphy after enterectomy, for intestinal anastomosis, for gastro-enterostomy, for lateral implantation as in ileo-colostomy, or for cholecystenterostomy. Last, but not least, after practice on models made of wash-leather and on the cadaver, I have proved its safety in actual practice in a case of a man aged sixty-five, suffering from stricture of the ascending colon producing intestinal obstruction, where I shortcircuited the obstruction by connecting the lower end of the ileum to the colon by means of the oval modification of the cotton-reel shaped bone tube, the patient making an uninterrupted recovery, and having his bowels moved 
regularly by the rectum daily subsequent to the second day after operation. I have to thank Dr. McGregor Young for his kindness in making me the diagrams, and Messrs. Maw and Thompson for the admirable manner in which they have manufactured the tubes.

(For report of the discussion on this paper, see ' Proceedings of the Royal Medical and Chirurgical Society,' Third Series, vol. iv, p. 16.)

\section{DESCRIPTION OF PLATE VI.}

Two Cases of Pylorectomy and One of Jejunostomy

(Mr. A. W. Mayo Robson, F.R.C.S.).

Fig. 1.-The continuous serous suture is applied, a third to half an inch from the margins of the openings in the viscera, first posteriorly. The ends are left long, with the needle still threaded, in order to complete the suture after the tube has been introduced and the marginal suture completed.

Fra. 2.-The continuous marginal suture is applied first around the posterior margins of the openings in front of the serous sutures Both ends of the suture are left long, the needle being threaded still at one end, to complete the closure as soon as the bone tube is in position.

Fig. 3. -The posterior portions of the serous and marginal suture already inserted having been drawn tight, the bone tube is placed in situ, and the continuous marginal suture is proceeded with in front of the tube.

Fra. 4.-The continuous marginal suture having been completed, it is drawn tight and tied, thus making the margins of the visceral openings embrace the tube all round, and effectually closing the viscera. The serous suture is then continued round in front, a third to half an inch from the margins. (Above, the serous suture is shown in situ before being drawn tight.)

Fra. 5.-The serous suture is then tightened and cut off short, leaving a third to half an inch of serous surfaces in apposition. (The sutures are buried and cannot be seen. The tube is shown in dotted lines.) 
Plate VI.

Med. Chir. Trans Vo1 LXXV

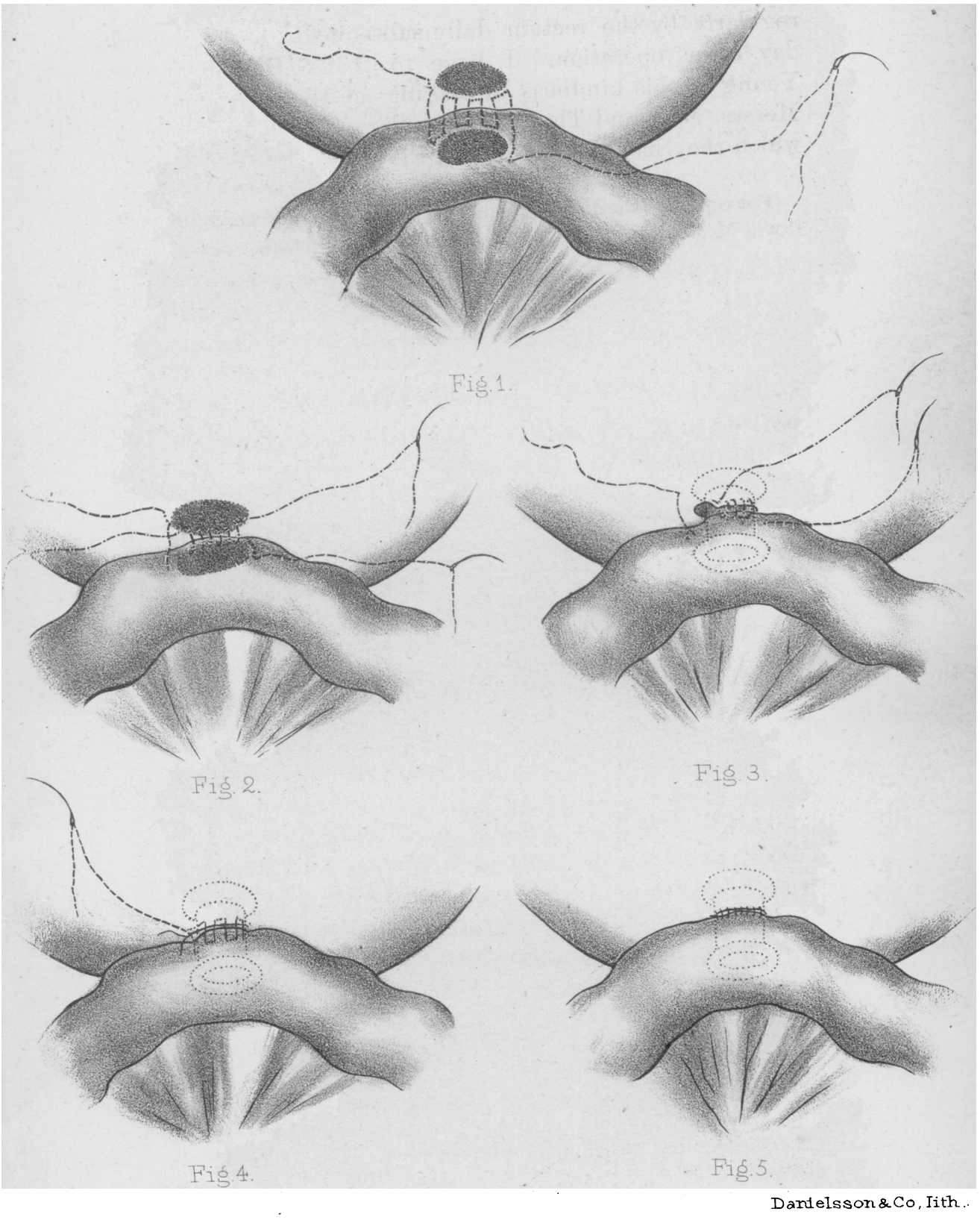

